\title{
A dynamical version of the Mordell-Lang conjecture for the additive group
}

\author{
D. Ghioca and T. J. Tucker
}

\begin{abstract}
We prove a dynamical version of the Mordell-Lang conjecture in the context of Drinfeld modules. We use analytic methods similar to those employed by Skolem, Chabauty, and Coleman for studying diophantine equations.
\end{abstract}

\section{Introduction}

Faltings proved the Mordell-Lang conjecture in the following form (see [Fal94]).

Theorem 1.1 (Faltings). Let $G$ be an abelian variety defined over the field of complex numbers $\mathbb{C}$. Let $X \subset G$ be a closed subvariety and $\Gamma \subset G(\mathbb{C})$ a finitely generated subgroup of $G(\mathbb{C})$. Then $X(\mathbb{C}) \cap \Gamma$ is a finite union of cosets of subgroups of $\Gamma$.

In particular, Theorem 1.1 says that an irreducible subvariety $X$ of an abelian variety $G$ only has a Zariski dense intersection with a finitely generated subgroup of $G(\mathbb{C})$ if $X$ is a translate of an algebraic subgroup of $G$. We also note that Faltings result was generalized to semiabelian varieties $G$ by Vojta (see [Voj96]), and then to finite rank subgroups $\Gamma$ of $G$ by McQuillan (see [McQ95]), while the function field case in characteristic $p$ was proved by Hrushovski (see [Hru96]).

If we try to formulate the Mordell-Lang conjecture in the context of algebraic subvarieties contained in a power of the additive group scheme $\mathbb{G}_{a}$, the conclusion is either false (in the characteristic zero case, as shown by the curve $y=x^{2}$ which has an infinite intersection with the finitely generated subgroup $\mathbb{Z} \times \mathbb{Z}$, without being itself a translate of an algebraic subgroup of $\mathbb{G}_{a}^{2}$ ) or it is trivially true (in the characteristic $p>0$ case, because every finitely generated subgroup of a power of $\mathbb{G}_{a}$ is finite). Denis [Den92a] formulated a Mordell-Lang conjecture for powers of $\mathbb{G}_{a}$ in characteristic $p$ in the context of Drinfeld modules. Denis replaced the finitely generated subgroup from the usual Mordell-Lang statement with a finitely generated $\phi$-submodule, where $\phi$ is a Drinfeld module. He also strengthened the conclusion of the Mordell-Lang statement by requiring that the subgroups whose cosets are contained in the intersection of the algebraic variety with the finitely generated $\phi$-submodule actually be $\phi$-submodules. The first author proved several cases of the Denis-MordellLang conjecture in [Ghi05] and [Ghi06b].

In the present paper we investigate other cases of the Denis-Mordell-Lang conjecture through methods different from those employed in [Ghi05]. In particular, we prove the Denis-Mordell-Lang conjecture in the case where the finitely generated $\phi$-module is cyclic and the Drinfeld modules are defined over a field of transcendence degree equal to one (this is our Theorem 2.5). Note that [Ghi05] and [Ghi06b] treat only the case where the transcendence degree of the field of definition is greater than one. One of the methods employed in [Ghi05] (and whose outcome was later used in [Ghi06b])

Received 29 January 2007, accepted in final form 24 August 2007, published online 7 February 2008.

2000 Mathematics Subject Classification 11G09 (primary), 14K12, 37F10 (secondary).

Keywords: Drinfeld module, polynomial dynamics, Mordell-Lang conjecture.

The second author was partially supported by National Security Agency Grant 06G-067.

This journal is (c) Foundation Compositio Mathematica 2008. 
was specializations; hence, the necessity of dealing with fields of transcendence degree greater than one. By contrast, the techniques used in this paper are more akin to those used in treating diophantine problems over number fields (see [Cha41], [Col85], or [BS66, ch. 4.6], for example), where such specialization arguments are also not available. So, making a parallel between the classical MordellLang conjecture and the Denis-Mordell-Lang conjecture, we might say that [Ghi05] and [Ghi06b] deal with the 'function field case', while our present paper deals with the 'number field case' of the Denis conjecture. Moreover, using specializations (as in [Hru98] and [Ghi05]), our Theorem 2.5 can be extended to Drinfeld modules defined over fields of arbitrary finite transcendence degree.

We also note that recently there has been significant progress on establishing additional links between classical diophantine results over number fields and similar statements for Drinfeld modules. The first author proved in [Ghi06a] an equidistribution statement for torsion points of a Drinfeld module, which is similar to the equidistribution statement established by Szpiro-UllmoZhang [SUZ97] (which was later extended by Zhang [Zha98] to a full proof of the famous Bogomolov conjecture). Bosser [Bos99] proved a lower bound for linear forms in logarithms at an infinite place associated to a Drinfeld module (similar to the classical result obtained by Baker [Bak75] for usual logarithms, by David [Dav95] for elliptic logarithms, or by Hirata-Khono [Hir91] for logarithms on arbitrary commutative algebraic groups defined over number fields). Bosser's result was used by the present authors in [GT] to establish certain equidistribution and integrality statements for Drinfeld modules. Moreover, Bosser's result is quite possibly true also for linear forms in logarithms at finite places for a Drinfeld module. Assuming that this last statement holds, the present authors proved in [GT07a] the analog of Siegel's theorem for finitely generated $\phi$-submodules. We believe that our present paper provides an additional proof of the fact that the Drinfeld modules represent the right arithmetic analog in characteristic $p$ for abelian varieties in characteristic zero.

The idea behind the proof of our Theorem 2.5 can be explained quite simply. Assuming that an affine variety $V \subset \mathbb{G}_{a}^{g}$ has infinitely many points in common with a cyclic $\phi$-submodule $\Gamma$, we can then find a suitable submodule $\Gamma_{0} \subset \Gamma$ whose coset lies in $V$. Indeed, applying the logarithmic map (associated to a suitable place $v$ ) to $\Gamma_{0}$ yields a line in the vector space $\mathbb{C}_{v}^{g}$. Each polynomial $f$ that vanishes on $V$, then gives rise to an analytic function $F$ on this line (by composing with the exponential function). Because we assumed that there are infinitely many points in $V \cap \Gamma$, the zeros of $F$ must have an accumulation point on this line, which means that $F$ vanishes identically on the line. This means that there is an entire translate of $\Gamma_{0}$ contained in the zero locus of $f$. The inspiration for this idea comes from the method employed by Chabauty in [Cha41] (and later refined by Coleman in [Col85]) to study the intersection of a curve $C$ of genus $g$, embedded in its Jacobian $J$, with a finitely generated subgroup of $J$ of rank less than $g$. Our technique also bears a resemblance to Skolem's method for treating diophantine equations (see [BS66, ch. 4.6]).

Alternatively, our results can be interpreted purely from the point of view of polynomial dynamics, as we describe the intersection of affine varieties with the iterates of a point in the affine space under polynomial actions on each coordinate. In this paper we treat the case of affine varieties embedded in $\mathbb{G}_{a}^{g}$, while the polynomial action (on each coordinate of $\mathbb{G}_{a}^{g}$ ) will always be given by Drinfeld modules. The more general problem of studying intersections of affine varieties with the iterates of a point in affine space under polynomial actions over number fields or function fields appears to be quite difficult. However, recently the present authors were able to extend in characteristic zero the logarithmic approach to polynomial dynamics from this paper (see [GT07b]). In [GTZ], Ghioca-Tucker-Zieve described the intersection between any line in the affine plane and an orbit of a point in $\mathbb{C}$ under polynomial actions on each coordinate of $\mathbb{A}^{2}$. On the other hand, the question of describing the intersection of a subvariety of a semiabelian variety $S$ with an orbit of a point in $S$ under an endomorphism of $S$ was completely settled (see [Voj96], [McQ95] and [GT07c]). We refer the reader to a recent paper by Zhang [Zha06] for a number of algebraic dynamical conjectures that 


\section{GHioca And T. J. TUCKer}

would generalize well-known arithmetic theorems for semiabelian varieties. Although [Zha06] does not contain a dynamical analog of the Mordell-Lang conjecture, Zhang has indicated to us that it might be reasonable to conjecture that if $\psi: Y \rightarrow Y$ is a suitable morphism of a projective variety $Y$ (one that is 'polarized', to use the terminology of [Zha06]), then the intersection of the $\psi$-orbit of a point $\beta$ with a subvariety $V$ must be finite if $V$ does not contain a positive dimensional periodic subvariety.

We briefly sketch the plan of our paper. In $\S 2$ we set the notation, describe the Denis-MordellLang conjecture, and then state our main result. In $\S 3$ we prove this main result (Theorem 2.5), while in $\S 4$ we prove a couple of extensions of it (Theorems 4.1 and 4.2 ).

\section{Notation and statement of our main result}

All subvarieties appearing in this paper are closed.

\subsection{Drinfeld modules}

We begin by defining a Drinfeld module. Let $p$ be a prime and let $q$ be a power of $p$. Let $A:=\mathbb{F}_{q}[t]$, let $K$ be a finite field extension of $\mathbb{F}_{q}(t)$, and let $\bar{K}$ be an algebraic closure of $K$. Let $K^{\text {sep }}$ be the separable closure of $K$ inside $\bar{K}$. We let $\tau$ be the Frobenius on $\mathbb{F}_{q}$, and we extend its action on $\bar{K}$. Let $K\{\tau\}$ be the ring of polynomials in $\tau$ with coefficients from $K$ (the addition is the usual addition, while the multiplication is the composition of functions).

A Drinfeld module is a morphism $\phi: A \rightarrow K\{\tau\}$ for which the coefficient of $\tau^{0}$ in $\phi(a)=: \phi_{a}$ is $a$ for every $a \in A$, and there exists $a \in A$ such that $\phi_{a} \neq a \tau^{0}$. The definition given here represents what Goss [Gos96] calls a Drinfeld module of 'generic characteristic'.

We note that usually, in the definition of a Drinfeld module, $A$ is the ring of functions defined on a projective nonsingular curve $C$, regular away from a closed point $\eta \in C$. For our definition of a Drinfeld module, $C=\mathbb{P}_{\mathbb{F}_{q}}^{1}$ and $\eta$ is the usual point at infinity on $\mathbb{P}^{1}$. On the other hand, every ring of regular functions $A$ as above contains $\mathbb{F}_{q}[t]$ as a subring, where $t$ is a nonconstant function in $A$.

For every field extension $K \subset L$, the Drinfeld module $\phi$ induces an action on $\mathbb{G}_{a}(L)$ by $a * x:=$ $\phi_{a}(x)$, for each $a \in A$. We call $\phi$-submodules subgroups of $\mathbb{G}_{a}(\bar{K})$ which are invariant under the action of $\phi$. We define the rank of a $\phi$-submodule $\Gamma$ be

$$
\operatorname{dim}_{\mathbb{F}_{q}(t)} \Gamma \otimes_{A} \mathbb{F}_{q}(t) .
$$

If $\phi_{1}: A \rightarrow K\{\tau\}, \ldots, \phi_{g}: A \rightarrow K\{\tau\}$ are Drinfeld modules, then $\left(\phi_{1}, \ldots, \phi_{g}\right)$ acts on $\mathbb{G}_{a}^{g}$ coordinate-wise (i.e. $\phi_{i}$ acts on the $i$ th coordinate). We define as above the notion of a $\left(\phi_{1}, \ldots, \phi_{g}\right)$ submodule of $\mathbb{G}_{a}^{g}$ and the same for its rank.

A point $\alpha$ is torsion for the Drinfeld module action if and only if there exists $Q \in A \backslash\{0\}$ such that $\phi_{Q}(\alpha)=0$. The set of all torsion points is denoted by $\phi_{\text {tor }}$.

\subsection{Valuations}

Let $M_{\mathbb{F}_{q}(t)}$ be the set of places on $\mathbb{F}_{q}(t)$. We denote by $v_{\infty}$ the place in $M_{\mathbb{F}_{q}(t)}$ such that $v_{\infty}(f / g)=$ $\operatorname{deg}(g)-\operatorname{deg}(f)$ for every nonzero $f, g \in A=\mathbb{F}_{q}[t]$. We let $M_{K}$ be the set of valuations on $K$. Then $M_{K}$ is a set of valuations which satisfies a product formula (see [Ser97, ch. 2]). Thus:

- for each nonzero $x \in K$, there are finitely many $v \in M_{K}$ such that $|x|_{v} \neq 1$; and

- for each nonzero $x \in K$, we have $\prod_{v \in M_{K}}|x|_{v}=1$.

Definition 2.1. Each place in $M_{K}$ which lies over $v_{\infty}$ is called an infinite place. Each place in $M_{K}$ which does not lie over $v_{\infty}$ is called a finite place. 
By abuse of notation, we let $\infty \in M_{K}$ denote any place extending the place $v_{\infty}$.

For $v \in M_{K}$ we let $K_{v}$ be the completion of $K$ with respect to $v$. Let $\mathbb{C}_{v}$ be the completion of an algebraic closure of $K_{v}$. Then $|\cdot|_{v}$ extends to a unique absolute value on all of $\mathbb{C}_{v}$. We fix an embedding of $i: \bar{K} \longrightarrow \mathbb{C}_{v}$. For $x \in \bar{K}$, we denote $|i(x)|_{v}$ simply as $|x|_{v}$, by abuse of notation.

\subsection{Logarithms and exponentials associated to a Drinfeld module}

Let $v \in M_{K}$. According to [Gos96, Proposition 4.6.7], there exists a unique formal power series $\exp _{\phi, v} \in \mathbb{C}_{v}\{\tau\}$ such that for every $a \in A$, we have

$$
\phi_{a}=\exp _{\phi, v} a \exp _{\phi, v}^{-1} .
$$

In addition, the coefficient of the linear term in $\exp _{\phi, v}(X)$ equals one. We let $\log _{\phi, v}$ be the formal power series $\exp _{\phi, v}^{-1}$, which is the inverse of $\exp _{\phi, v}$.

If $v=\infty$ is an infinite place, then $\exp _{\phi, \infty}(x)$ is convergent for all $x \in \mathbb{C}_{\infty}$ (see [Gos96, Theorem 4.6.9]). There exists a sufficiently small ball $B_{\infty}$ centered at the origin such that $\exp _{\phi, \infty}$ is an isometry on $B_{\infty}$ (see [GT, Lemma 3.6]). Hence, $\log _{\phi, \infty}$ is convergent on $B_{\infty}$. Moreover, the restriction of $\log _{\phi, \infty}$ on $B_{\infty}$ is an analytic isometry (see also [Gos96, Proposition 4.14.2]).

If $v$ is a finite place, then $\exp _{\phi, v}$ is convergent on a sufficiently small ball $B_{v} \subset \mathbb{C}_{v}$ (this follows in an identical manner to the proof of the analyticity of $\exp _{\phi, \infty}$ from [Gos96, Theorem 4.6.9]). Similarly as in the above paragraph, at the expense of replacing $B_{v}$ by a smaller ball, we may assume that $\exp _{\phi, v}$ is an isometry on $B_{v}$. Hence, $\log _{\phi, v}$ is also an analytic isometry on $B_{v}$.

For every place $v \in M_{K}$, for every $x \in B_{v}$, and for every polynomial $a \in A$, we have (see (2.1.1))

$$
a \log _{\phi, v}(x)=\log _{\phi, v}\left(\phi_{a}(x)\right) \text { and } \exp _{\phi, v}(a x)=\phi_{a}\left(\exp _{\phi, v}(x)\right) .
$$

By abuse of language, $\exp _{\phi, \infty}$ and $\exp _{\phi, v}$ will be called exponentials, while $\log _{\phi, \infty}$ and $\log _{\phi, v}$ will be called logarithms.

\subsection{Integrality and reduction}

Definition 2.2. A Drinfeld module $\phi$ has good reduction at a place $v$ if for each nonzero $a \in A$, all coefficients of $\phi_{a}$ are $v$-adic integers and the leading coefficient of $\phi_{a}$ is a $v$-adic unit. If $\phi$ does not have good reduction at $v$, then we say that $\phi$ has bad reduction at $v$.

It is immediate to see that $\phi$ has good reduction at $v$ if and only if all coefficients of $\phi_{t}$ are $v$-adic integers, while the leading coefficient of $\phi_{t}$ is a $v$-adic unit. All infinite places of $K$ are places of bad reduction for $\phi$. We also note that our definition for places of good reduction is not invariant under isomorphisms of Drinfeld modules.

\subsection{The Denis-Mordell-Lang conjecture}

Let $g$ be a positive integer.

Definition 2.3. Let $\phi_{1}: A \rightarrow K\{\tau\}, \ldots, \phi_{g}: A \rightarrow K\{\tau\}$ be Drinfeld modules. An algebraic $\left(\phi_{1}, \ldots, \phi_{g}\right)$-submodule of $\mathbb{G}_{a}^{g}$ is an irreducible algebraic subgroup of $\mathbb{G}_{a}^{g}$ invariant under the action of $\left(\phi_{1}, \ldots, \phi_{g}\right)$.

Denis proposed in [Den92a, Conjecture 2] the following problem, which we call the full DenisMordell-Lang conjecture because it asks for the description of the intersection of an affine variety with a finite rank $\phi$-module (as opposed to only a finitely generated $\phi$-module). Recall that a $\phi$ module $M$ is said to be a finite rank $\phi$-module if it contains a finitely generated $\phi$-submodule such that $M / M^{\prime}$ is a torsion $\phi$-module. 


\section{GHioca And T. J. TUCKer}

Conjecture 2.4 (The full Denis-Mordell-Lang conjecture). Let $\phi_{1}: A \rightarrow K\{\tau\}, \ldots, \phi_{g}: A \rightarrow$ $K\{\tau\}$ be Drinfeld modules. Let $V \subset \mathbb{G}_{a}^{g}$ be an affine variety defined over $\bar{K}$. Let $\Gamma$ be a finite rank $\left(\phi_{1}, \ldots, \phi_{g}\right)$-submodule of $\mathbb{G}_{a}^{g}(\bar{K})$. Then there exist algebraic $\left(\phi_{1}, \ldots, \phi_{g}\right)$-submodules $B_{1}, \ldots, B_{l}$ of $\mathbb{G}_{a}^{g}$ and there exist $\gamma_{1}, \ldots, \gamma_{l} \in \Gamma$ such that

$$
V(\bar{K}) \cap \Gamma=\bigcup_{i=1}^{l}\left(\gamma_{i}+B_{i}(\bar{K})\right) \cap \Gamma .
$$

In [Den92a], Denis showed that under certain natural Galois theoretical assumptions, Conjecture 2.4 would follow from the weaker conjecture which would describe the intersection of an affine variety with a finitely generated $\phi$-module.

Since then, Scanlon [Sca02] has proved Conjecture 2.4 in the case where $\Gamma$ is the product of the torsion submodules of each $\phi_{i}$, and the first author has worked out various other instances of Conjecture 2.4 in [Ghi05] and [Ghi06b]. We note that Denis posed his conjecture more generally for $t$-modules, which includes the case of products of distinct Drinfeld modules acting on $\mathbb{G}_{a}^{g}$.

For the sake of simplifying the notation, we denote by $\phi$ the action of $\left(\phi_{1}, \ldots, \phi_{g}\right)$ on $\mathbb{G}_{a}^{g}$. We also note that if $V$ is an irreducible affine subvariety of $\mathbb{G}_{a}^{g}$ which has a Zariski-dense intersection with a finite rank $\phi$-submodule $\Gamma$ of $\mathbb{G}_{a}^{g}$, then the Denis-Mordell-Lang conjecture predicts that $V$ is a translate of an algebraic $\phi$-submodule of $\mathbb{G}_{a}^{g}$ by a point in $\Gamma$. In particular, if $V$ is an irreducible affine curve, which is not a translate of an algebraic $\phi$-submodule, then its intersection with any finite rank $\phi$-submodule of $\mathbb{G}_{a}^{g}$ should be finite.

In [Ghi05], the first author studied the Denis-Mordell-Lang conjecture for Drinfeld modules whose field of definition (for their coefficients) is of transcendence degree at least equal to two over $\mathbb{F}_{p}$. The methods employed in [Ghi05] involve specializations, and so it was crucial for the $\phi$ there not to be isomorphic with a Drinfeld module defined over $\overline{\mathbb{F}_{q}(t)}$. In the present paper we study precisely this case left out in [Ghi05] and [Ghi06b]. Our methods depend crucially on the hypothesis that the transcendence degree of the field generated by the coefficients of $\phi_{i}$ is one, since we use the fact that at each place $v$, the number of residue classes in the ring of integers at $v$ is finite.

The main result of our paper is describing the intersection of an affine subvariety $V \subset \mathbb{G}_{a}^{g}$ with a cyclic $\phi$-submodule $\Gamma$ of $\mathbb{G}_{a}^{g}$ (i.e. $\Gamma$ is generated by a single element of $\mathbb{G}_{a}^{g}$ ).

Theorem 2.5. Let $K$ be a finite extension of $\mathbb{F}_{q}(t)$. Let $\phi_{1}: A \rightarrow K\{\tau\}, \ldots, \phi_{g}: A \rightarrow K\{\tau\}$ be Drinfeld modules. Let $\left(x_{1}, \ldots, x_{g}\right) \in \mathbb{G}_{a}^{g}(K)$ and let $\Gamma \subset \mathbb{G}_{a}^{g}(K)$ be the cyclic $\left(\phi_{1}, \ldots, \phi_{g}\right)$-submodule generated by $\left(x_{1}, \ldots, x_{g}\right)$. Let $V \subset \mathbb{G}_{a}^{g}$ be an affine subvariety defined over $K$. Then $V(K) \cap \Gamma$ is a finite union of cosets of $\left(\phi_{1}, \ldots, \phi_{g}\right)$-submodules of $\Gamma$. Moreover, each submodule of $\Gamma$ whose coset appears in the above intersection is of the form $B_{i}(K) \cap \Gamma$, where each $B_{i}$ is an algebraic $\left(\phi_{1}, \ldots, \phi_{g}\right)$-submodule of $\mathbb{G}_{a}^{g}$.

Using an idea from [Ghi06b], we are able to extend the above result to $\left(\phi_{1}, \ldots, \phi_{g}\right)$-submodules of rank one (see our Theorem 4.2) in the special case where $V$ is a curve.

\section{Proofs of our main results}

We continue with the notation from $\S 2$. Hence, $\phi_{1}, \ldots, \phi_{g}$ are Drinfeld modules. We denote by $\phi$ the action of $\left(\phi_{1}, \ldots, \phi_{g}\right)$ on $\mathbb{G}_{a}^{g}$. Also, let $\left(x_{1}, \ldots, x_{g}\right) \in \mathbb{G}_{a}^{g}(K)$ and let $\Gamma$ be the cyclic $\phi$-submodule of $\mathbb{G}_{a}^{g}(K)$ generated by $\left(x_{1}, \ldots, x_{g}\right)$. Unless otherwise stated, $V \subset \mathbb{G}_{a}^{g}$ is an affine subvariety defined over $K$.

We first prove an easy combinatorial result which we use in the proof of Theorem 2.5. 
Lemma 3.1. Let $\Gamma$ be a cyclic $\phi$-submodule of $\mathbb{G}_{a}^{g}(K)$. Let $\Gamma_{0}$ be a nontrivial $\phi$-submodule of $\Gamma$, and let $S \subset \Gamma$ be an infinite set. Suppose that for every infinite subset $S_{0} \subset S$, there exists a coset $C_{0}$ of $\Gamma_{0}$ such that $C_{0} \cap S_{0} \neq \emptyset$ and $C_{0} \subset S$. Then $S$ is a finite union of cosets of $\phi$-submodules of $\Gamma$.

Proof. Since $S$ is infinite, $\Gamma$ is infinite and thus torsion-free. Therefore, $\Gamma$ is an infinite cyclic $\phi$-module, which is isomorphic to $A$ (as a module over itself). Hence, via this isomorphism, $\Gamma_{0}$ is isomorphic to a nontrivial ideal $I$ of $A$. Since $A / I$ is finite (recall that $A=\mathbb{F}_{q}[t]$ ), there are finitely many cosets of $\Gamma_{0}$ in $\Gamma$. Thus, $S$ contains at most finitely many cosets of $\Gamma_{0}$.

Now, let $\left\{y_{i}+\Gamma_{0}\right\}_{i=1}^{\ell}$ be all of the cosets of $\Gamma_{0}$ that are contained in $S$. Suppose that

$$
S_{0}:=S \backslash \bigcup_{i=1}^{\ell}\left(y_{i}+\Gamma_{0}\right) \quad \text { is infinite. }
$$

Then using the hypotheses of Lemma 3.1 for $S_{0}$, we see that there is a coset of $\Gamma_{0}$ that is contained in $S$ but is not one of the cosets $\left(y_{i}+\Gamma_{0}\right)$ (because it has a nonempty intersection with $S_{0}$ ). This contradicts the fact that $\left\{y_{i}+\Gamma_{0}\right\}_{i=1}^{\ell}$ are all of the cosets of $\Gamma_{0}$ that are contained in $S$. Therefore, $S_{0}$ must be finite. Since any finite subset of $\Gamma$ is a finite union of cosets of the trivial submodule of $\Gamma$, this completes our proof.

We also use the following lemma in the proof of Theorem 2.5.

Lemma 3.2. Let $\theta: A \rightarrow K\{\tau\}$ and $\psi: A \rightarrow K\{\tau\}$ be Drinfeld modules. Let $v$ be a place of good reduction for $\theta$ and $\psi$. Let $x, y \in \mathbb{C}_{v}$. Let $0<r_{v}<1$ and let $B_{v}:=\left\{\left.z \in \mathbb{C}_{v}|| z\right|_{v}<r_{v}\right\}$ be a sufficiently small ball centered at the origin with the property that both $\log _{\theta, v}$ and $\log _{\psi, v}$ are analytic isometries on $B_{v}$. Then for all polynomials $P, Q \in A$ such that $\left(\theta_{P}(x), \psi_{P}(y)\right) \in B_{v} \times B_{v}$ and $\left(\theta_{Q}(x), \psi_{Q}(y)\right) \in B_{v} \times B_{v}$, we have

$$
\log _{\theta, v}\left(\theta_{P}(x)\right) \cdot \log _{\psi, v}\left(\psi_{Q}(y)\right)=\log _{\theta, v}\left(\theta_{Q}(x)\right) \cdot \log _{\psi, v}\left(\psi_{P}(y)\right) .
$$

Proof. Since $v$ is a place of good reduction for $\theta$, all of the coefficients of $\theta_{Q}$ are $v$-adic integers and, thus, $\left|\theta_{Q}\left(\theta_{P}(x)\right)\right|_{v} \leqslant\left|\theta_{P}(x)\right|_{v}<r_{v}$ (we use the fact that $\left|\theta_{P}(x)\right|_{v}<r_{v}<1$ and so each term of $\theta_{Q}\left(\theta_{P}(x)\right)$ has its absolute value at most equal to $\left.\left|\theta_{P}(x)\right|_{v}\right)$. Using (2.1.2), we conclude that

$$
Q \cdot \log _{\theta, v}\left(\theta_{P}(x)\right)=\log _{\theta, v}\left(\theta_{Q P}(x)\right)=\log _{\theta, v}\left(\theta_{P Q}(x)\right)=P \cdot \log _{\theta, v}\left(\theta_{Q}(x)\right) .
$$

Similarly we obtain that $Q \cdot \log _{\psi, v}\left(\psi_{P}(x)\right)=P \cdot \log _{\psi, v}\left(\psi_{Q}(x)\right)$. This concludes the proof of Lemma 3.2.

The following result is an immediate corollary of Lemma 3.2.

Corollary 3.3. With the notation as in Theorem 2.5, assume in addition that $x_{1} \notin\left(\phi_{1}\right)_{\text {tor }}$. Let $v$ be a place of good reduction for each $\phi_{i}$. Suppose that $B_{v}$ is a small ball (of radius less than one) centered at the origin such that each $\log _{\phi_{i}, v}$ is an analytic isometry on $B_{v}$. Then for each $i \in\{2, \ldots, g\}$, the fractions

$$
\lambda_{i}:=\frac{\log _{\phi_{i}, v}\left(\left(\phi_{i}\right)_{P}\left(x_{i}\right)\right)}{\log _{\phi_{1}, v}\left(\left(\phi_{1}\right)_{P}\left(x_{1}\right)\right)}
$$

are independent of the choice of the nonzero polynomial $P \in A$ for which $\phi_{P}\left(x_{1}, \ldots, x_{g}\right) \in B_{v}^{g}$.

The following simple result on zeros of analytic functions can be found in [Gos96, Proposition 2.1, p. 42]. We include a short proof for the sake of completeness.

Lemma 3.4. Let $F(z)=\sum_{i=0}^{\infty} a_{i} z^{i}$ be a power series with coefficients in $\mathbb{C}_{v}$ that is convergent in an open disc $B$ of positive radius around the point $z=0$. Suppose that $F$ is not the zero function. Then the zeros of $F$ in $B$ are isolated. 


\section{GHioca And T. J. TUCKer}

Proof. Let $w$ be a zero of $F$ in $B$. We may rewrite $F$ in terms of $(z-w)$ as a power series $F(z)=\sum_{i=1}^{\infty} b_{i}(z-w)^{i}$ that converges in a disc $B_{w}$ of positive radius around $w$. Let $m$ be the smallest index $n$ such that $b_{n} \neq 0$.

Because $F$ is convergent in $B_{w}$, then there exists a positive real number $r$ such that for all $n>m$, we have $\left|b_{n} / b_{m}\right|_{v}<r^{n-m}$. Then, for any $u \in B_{w}$ such that $0<|u-w|_{v}<1 / r$, we have $\left|b_{m}(u-w)^{m}\right|_{v}>\left|b_{n}(u-w)^{n}\right|_{v}$ for all $n>m$. Hence, $|F(u)|_{v}=\left|b_{m}(u-w)^{m}\right|_{v} \neq 0$. Thus, $F(u) \neq 0$ and so $F$ has no zeros other than $w$ in a nonempty open disc around $w$.

We are ready to prove Theorem 2.5.

Proof of Theorem 2.5. We begin by showing that the 'moreover' clause follows from the main statement. Indeed, if $(b+H) \subset V(K)$ is a coset of a submodule $H$ of $\Gamma$, then $(b+D) \subset V$, where $D$ is the Zariski closure of $H$. Since $H$ is a $\phi$-submodule, each $D$ is mapped into itself by the $\phi$-action. Hence, it is a finite union of translates $\left(b_{i}+B_{i}\right)$ of algebraic $\phi$-submodules $B_{i}$ of $\mathbb{G}_{a}^{g}$ (see [Den92a, Lemme 4]). Therefore, we may write $(b+H) \subset \bigcup_{i}\left(c_{i}+\left(B_{i}(K) \cap \Gamma\right)\right) \subset V(K)$, where $c_{i} \in\left(b+b_{i}+B_{i}(K)\right) \cap \Gamma$ for each $i$. Thus, the 'moreover' clause in Theorem 2.5 is a consequence of the main statement of the theorem.

We may assume that $V(K) \cap \Gamma$ is infinite (otherwise the conclusion of Theorem 2.5 is obviously satisfied). Assuming that $V(K) \cap \Gamma$ is infinite, we show that there exists a nontrivial $\phi$-submodule $\Gamma_{0} \subset \Gamma$ such that each infinite subset of points $S_{0}$ in $V(K) \cap \Gamma$ has a nonempty intersection with a coset $C_{0}$ of $\Gamma_{0}$ and, moreover, $C_{0} \subset V(K) \cap \Gamma$. Theorem 2.5 will then follow immediately from Lemma 3.1.

First we observe that $\Gamma$ is not a torsion $\phi$-submodule. Otherwise $\Gamma$ is finite, contradicting our assumption that $V(K) \cap \Gamma$ is infinite. Hence, from now on, we assume (without loss of generality) that $x_{1}$ is not a torsion point for $\phi_{1}$. of $V$.

We fix a finite set of polynomials $\left\{f_{j}\right\}_{j=1}^{\ell} \subset K\left[X_{1}, \ldots, X_{g}\right]$ which generate the vanishing ideal

Let $v \in M_{K}$ be a place of $K$ which is of good reduction for all $\phi_{i}$ (for $1 \leqslant i \leqslant g$ ). In addition, we assume each $x_{i}$ is integral at $v$ (for $1 \leqslant i \leqslant g$ ). Then for each $P \in A$, we have

$$
\phi_{P}\left(x_{1}, \ldots, x_{g}\right) \in \mathbb{G}_{a}^{g}\left(\mathfrak{o}_{v}\right),
$$

where $\mathfrak{o}_{v}$ is the ring of $v$-adic integers in $K_{v}$ (the completion of $K$ at $v$ ). Because $\mathfrak{o}_{v}$ is a compact space (we use the fact that $K$ is a function field of transcendence degree 1 and thus has a finite residue field at $v$ ), we conclude that every infinite sequence of points $\phi_{P}\left(x_{1}, \ldots, x_{g}\right) \in V(K) \cap \Gamma$ contains a convergent subsequence in $\mathfrak{o}_{v}^{g}$. Using Lemma 3.1, it suffices to show that there exists a nontrivial $\phi$-submodule $\Gamma_{0} \subset \Gamma$ such that every convergent sequence of points in $V(K) \cap \Gamma$ has a nonempty intersection with a coset $C_{0}$ of $\Gamma_{0}$ and, moreover, $C_{0} \subset V(K) \cap \Gamma$.

Now, let $S_{0}$ be an infinite subsequence of distinct points in $V(K) \cap \Gamma$ which converges $v$-adically to $\left(x_{0,1}, \ldots, x_{0, g}\right) \in \mathfrak{o}_{v}^{g}$, let $0<r_{v}<1$, and let $B_{v}:=\left\{\left.z \in \mathbb{C}_{v}|| z\right|_{v}<r_{v}\right\}$ be a small ball centered at the origin on which each of the logarithmic functions $\log _{\phi_{i}, v}$ is an analytic isometry (for $1 \leqslant i \leqslant g$ ). Since $\left(x_{0,1}, \ldots, x_{0, g}\right)$ is the limit point for $S_{0}$, there exists a $d \in A$ and an infinite subsequence $\left\{\phi_{d+P_{n}}\right\}_{n \geqslant 0} \subset S_{0}$ (with $P_{n}=0$ if and only if $n=0$ ), such that for each $n \geqslant 0$, we have

$$
\left|\left(\phi_{i}\right)_{d+P_{n}}\left(x_{i}\right)-x_{0, i}\right|_{v}<\frac{r_{v}}{2} \text { for each } 1 \leqslant i \leqslant g .
$$

We show that there exists an algebraic group $Y_{0}$, independent of $S_{0}$ and invariant under $\phi$, such that $\phi_{d}\left(x_{1}, \ldots, x_{g}\right)+Y_{0}$ is a subvariety of $V$ containing $\phi_{d+P_{n}}\left(x_{1}, \ldots, x_{g}\right)$ for all $P_{n}$. Thus, the submodule $\Gamma_{0}:=Y_{0}(K) \cap \Gamma$ will satisfy the hypothesis of Lemma 3.1 for the infinite subset $V(K) \cap \Gamma \subset \Gamma$; this will yield the conclusion of Theorem 2.5. 
Using (3.4.1) for $n=0$ (we recall that $P_{0}=0$ ), and then for arbitrary $n$, we see that

$$
\left|\left(\phi_{i}\right)_{P_{n}}\left(x_{i}\right)\right|_{v}<\frac{r_{v}}{2} \text { for each } 1 \leqslant i \leqslant g .
$$

Hence, $\log _{\phi_{i}, v}$ is well defined at $\left(\phi_{i}\right)_{P_{n}}\left(x_{i}\right)$ for each $i \in\{1, \ldots, g\}$ and for each $n \geqslant 1$. Moreover, the fact that $\left(\left(\phi_{i}\right)_{P_{n}+d}\left(x_{i}\right)\right)_{n \geqslant 1}$ converges to a point in $\mathfrak{o}_{v}$ means that $\left(\left(\phi_{i}\right)_{P_{n}}\left(x_{i}\right)\right)_{n \geqslant 1}$ converges to a point which is contained in $B_{v}$ (see (3.4.2)).

Without loss of generality, we may assume

$$
\left|\log _{\phi_{1}, v}\left(\left(\phi_{1}\right)_{P_{1}}\left(x_{1}\right)\right)\right|_{v}=\max _{i=1}^{g}\left|\log _{\phi_{i}, v}\left(\left(\phi_{i}\right)_{P_{1}}\left(x_{i}\right)\right)\right|_{v} .
$$

In (3.4.3), we used the fact that the maximum cannot be attained at a torsion point $x_{i}$, because the logarithm vanishes precisely on the torsion points (actually, the only torsion point contained in $B_{v}$ is zero because $\log _{\phi_{i}, v}$ is an analytic isometry on $B_{v}$ for each $i$ ).

Using the result of Corollary 3.3, we conclude that for each $i \in\{2, \ldots, g\}$, the following fraction is independent of $n$ and of the sequence $\left\{P_{n}\right\}_{n}$ :

$$
\lambda_{i}:=\frac{\log _{\phi_{i}, v}\left(\left(\phi_{i}\right)_{P_{n}}\left(x_{i}\right)\right)}{\log _{\phi_{1}, v}\left(\left(\phi_{1}\right)_{P_{n}}\left(x_{1}\right)\right)} .
$$

Note that since $x_{1}$ is not a torsion point for $\phi_{1}$, the denominator of $\lambda_{i}$ in (3.4.4) is nonzero. Owing to (3.4.3), we may conclude that $\left|\lambda_{i}\right|_{v} \leqslant 1$ for each $i$.

The fact that $\lambda_{i}$ is independent of the sequence $\left\{P_{n}\right\}_{n \geqslant 1}$ will be used later to show that the $\phi$-submodule $\Gamma_{0}$ that we construct is independent of the sequence $\left\{P_{n}\right\}_{n} \geqslant 1$.

For each $n \geqslant 1$ and each $2 \leqslant i \leqslant g$, we have

$$
\log _{\phi_{i}, v}\left(\left(\phi_{i}\right)_{P_{n}}\left(x_{i}\right)\right)=\lambda_{i} \cdot \log _{\phi_{1}, v}\left(\left(\phi_{1}\right)_{P_{n}}\left(x_{1}\right)\right) .
$$

For each $i$, applying the exponential function $\exp _{\phi_{i}, v}$ to both sides of (3.4.5) yields

$$
\left(\phi_{i}\right)_{P_{n}}\left(x_{i}\right)=\exp _{\phi_{i}, v}\left(\lambda_{i} \cdot \log _{\phi_{1}, v}\left(\left(\phi_{1}\right)_{P_{n}}\left(x_{1}\right)\right)\right) .
$$

Since $\phi_{d+P_{n}}\left(x_{1}, \ldots, x_{g}\right) \in V(K)$, for each $j \in\{1, \ldots, \ell\}$ we have

$$
f_{j}\left(\phi_{d+P_{n}}\left(x_{1}, \ldots, x_{g}\right)\right)=0 \text { for each } n .
$$

For each $j \in\{1, \ldots, \ell\}$ we let $f_{d, j} \in K\left[X_{1}, \ldots, X_{g}\right]$ be defined by

$$
f_{d, j}\left(X_{1}, \ldots, X_{g}\right):=f_{j}\left(\phi_{d}\left(x_{1}, \ldots, x_{g}\right)+\left(X_{1}, \ldots, X_{g}\right)\right) .
$$

We let $V_{d} \subset \mathbb{G}_{a}^{g}$ be the affine subvariety defined by the equations

$$
f_{d, j}\left(X_{1}, \ldots, X_{g}\right)=0 \text { for each } j \in\{1, \ldots, \ell\} .
$$

Using (3.4.7) and (3.4.8), we see that for each $j \in\{1, \ldots, \ell\}$ we have

$$
f_{d, j}\left(\phi_{P_{n}}\left(x_{1}, \ldots, x_{g}\right)\right)=0
$$

for each $n$, and so

$$
\phi_{P_{n}}\left(x_{1}, \ldots, x_{g}\right) \in V_{d}(K)
$$

For each $j \in\{1, \ldots, \ell\}$, we let $F_{d, j}(u)$ be the analytic function defined on $B_{v}$ by

$$
F_{d, j}(u):=f_{d, j}\left(u, \exp _{\phi_{2}, v}\left(\lambda_{2} \log _{\phi_{1}, v}(u)\right), \ldots, \exp _{\phi_{g}, v}\left(\lambda_{g} \log _{\phi_{1}, v}(u)\right)\right) .
$$

We note, because of (3.4.3) and the fact that $\log _{\phi_{1}, v}$ is an analytic isometry on $B_{v}$ that for each $u \in B_{v}$ we have

$$
\left|\lambda_{i} \cdot \log _{\phi_{1}, v}(u)\right|_{v}=\left|\lambda_{i}\right|_{v} \cdot\left|\log _{\phi_{1}, v}(u)\right|_{v} \leqslant|u|_{v}<r_{v}
$$




\section{Ghioca And T. J. Tucker}

Equation (3.4.11) shows that $\lambda_{i} \cdot \log _{\phi_{1}, v}(u) \in B_{v}$, and so $\exp _{\phi_{i}, v}\left(\lambda_{i} \cdot \log _{\phi_{1}, v}(u)\right)$ is well defined.

Using (3.4.6) and (3.4.9) we obtain that, for every $n \geqslant 1$, we have

$$
F_{d, j}\left(\left(\phi_{1}\right)_{P_{n}}\left(x_{1}\right)\right)=0 .
$$

Thus, $\left(\left(\phi_{1}\right)_{P_{n}}\left(x_{1}\right)\right)_{n \geqslant 1}$ is a sequence of zeros for the analytic function $F_{d, j}$ which has an accumulation point in $B_{v}$. Lemma 3.4 then implies that $F_{d, j}=0$, and so, for each $j \in\{1, \ldots, \ell\}$, we have

$$
f_{d, j}\left(u, \exp _{\phi_{2}, v}\left(\lambda_{2} \log _{\phi_{1}, v}(u)\right), \ldots, \exp _{\phi_{g}, v}\left(\lambda_{g} \log _{\phi_{1}, v}(u)\right)\right)=0 .
$$

For each $u \in B_{v}$, we let

$$
Z_{u}:=\left(u, \exp _{\phi_{2}, v}\left(\lambda_{2} \log _{\phi_{1}, v}(u)\right), \ldots, \exp _{\phi_{g}, v}\left(\lambda_{g} \log _{\phi_{1}, v}(u)\right)\right) \in \mathbb{G}_{a}^{g}\left(\mathbb{C}_{v}\right) .
$$

Then (3.4.13) implies that

$$
Z_{u} \in V_{d} \text { for each } u \in B_{v} .
$$

Let $Y_{0}$ be the Zariski closure of $\left\{Z_{u}\right\}_{u \in B_{v}}$. Then $Y_{0} \subset V_{d}$. Note that $Y_{0}$ is independent of the sequence $\left\{P_{n}\right\}_{n}$ (because the $\lambda_{i}$ are independent of the sequence $\left\{P_{n}\right\}_{n}$, according to Corollary 3.3).

We claim that for each $u \in B_{v}$ and for each $P \in A$, we have

$$
\phi_{P}\left(Z_{u}\right)=Z_{\left(\phi_{1}\right)_{P}(u)} \text {. }
$$

Note that for each $u \in B_{v}$, then also $\left(\phi_{1}\right)_{P}(u) \in B_{v}$ for each $P \in A$, because each coefficient of $\phi_{1}$ is a $v$-adic integer. To see that (3.4.15) holds, we use (2.1.2), which implies that for each $i \in\{2, \ldots, g\}$ we have

$$
\begin{aligned}
\exp _{\phi_{i}, v}\left(\lambda_{i} \log _{\phi_{1}, v}\left(\left(\phi_{1}\right)_{P}(u)\right)\right) & =\exp _{\phi_{i}, v}\left(\lambda_{i} \cdot P \cdot \log _{\phi_{1}, v}(u)\right) \\
& =\exp _{\phi_{i}, v}\left(P \cdot \lambda_{i} \log _{\phi_{1}, v}(u)\right) \\
& =\left(\phi_{i}\right)_{P}\left(\exp _{\phi_{i}, v}\left(\lambda_{i} \log _{\phi_{1}, v}(u)\right)\right) .
\end{aligned}
$$

Hence, (3.4.15) holds, and so $Y_{0}$ is invariant under $\phi$. Furthermore, since all of the $\exp _{\phi_{i}, v}$ and $\log _{\phi_{i}, v}$ are additive functions, we have $Z_{u_{1}+u_{2}}=Z_{u_{1}}+Z_{u_{2}}$ for every $u_{1}, u_{2} \in B_{v}$. Hence, $Y_{0}$ is an algebraic group, which is also a $\phi$-submodule of $\mathbb{G}_{a}^{g}$. Moreover, $Y_{0}$ is defined independently of $\Gamma$.

Let $\Gamma_{0}:=Y_{0}(K) \cap \Gamma$. Because $Y_{0}$ is invariant under $\phi$, then $\Gamma_{0}$ is a submodule of $\Gamma$. Because $Y_{0} \subset V_{d}$, it follows that the translate $\phi_{d}\left(x_{1}, \ldots, x_{g}\right)+Y_{0}$ is a subvariety of $V$ which contains all $\left\{\phi_{d+P_{n}}\left(x_{1}, \ldots, x_{g}\right)\right\}_{n}$. In particular, the (infinite) translate $C_{0}$ of $\Gamma_{0}$ by $\phi_{d}\left(x_{1}, \ldots, x_{g}\right)$ is contained in $V(K) \cap \Gamma$. Hence, every infinite sequence of points in $V(K) \cap \Gamma$ has a nontrivial intersection with a coset $C_{0}$ of (the nontrivial $\phi$-submodule) $\Gamma_{0}$ and, moreover, $C_{0} \subset V(K) \cap \Gamma$. Applying Lemma 3.1 thus finishes the proof of Theorem 2.5.

In the course of our proof of Theorem 2.5 we also proved the following statement.

TheOrem 3.5. Let $\Gamma$ be an infinite cyclic $\phi$-submodule of $\mathbb{G}_{a}^{g}$. Then there exists an infinite $\phi$-submodule $\Gamma_{0} \subset \Gamma$ such that for every affine subvariety $V \subset \mathbb{G}_{a}^{g}$, if $V(\bar{K}) \cap \Gamma$ is infinite, then $V(\bar{K}) \cap \Gamma$ contains a coset of $\Gamma_{0}$.

Proof. Let $v$ be a place of good reduction for $\phi$; in addition, we assume that the points in $\Gamma$ are $v$-adic integers. Suppose that $V(\bar{K}) \cap \Gamma$ is infinite. As shown in the proof of Theorem 2.5, there exists a positive-dimensional algebraic group $Y_{0}$, invariant under $\phi$, and depending only on $\Gamma$ and $v$ (but not on $V$ ), such that a translate of $Y_{0}$ by a point in $\Gamma$ lies in $V$. Moreover, $\Gamma_{0}:=Y_{0}(\bar{K}) \cap \Gamma$ is infinite. Hence, $\Gamma_{0}$ satisfies the conclusion of Theorem 3.5.

\section{Further extensions}

We continue with the notation from $\S 3: \phi_{1}, \ldots, \phi_{g}$ are Drinfeld modules. As usual, we denote by $\phi$ the action of $\left(\phi_{1}, \ldots, \phi_{g}\right)$ on $\mathbb{G}_{a}^{g}$. First we prove the following consequence of Theorem 2.5. 
Theorem 4.1. Let $V \subset \mathbb{G}_{a}^{g}$ be an affine subvariety defined over $K$. Let $\Gamma \subset \mathbb{G}_{a}^{g}(K)$ be a finitely generated $\phi$-submodule of rank one. Then $V(K) \cap \Gamma$ is a finite union of cosets of $\phi$-submodules of $\Gamma$ of the form $B_{i}(K) \cap \Gamma$, where each $B_{i}$ is an algebraic $\phi$-submodule of $\mathbb{G}_{a}^{g}$. In particular, if $V$ is an irreducible curve which is not a translate of an algebraic $\phi$-submodule, then $V(K) \cap \Gamma$ is finite.

Proof. Since $A=\mathbb{F}_{q}[t]$ is a principal ideal domain, $\Gamma$ is the direct sum of its finite torsion submodule $\Gamma_{\text {tor }}$ and a free submodule $\Gamma_{1}$, which is cyclic because $\Gamma$ has rank one. Therefore,

$$
\Gamma=\bigcup_{\gamma \in \Gamma_{\text {tor }}} \gamma+\Gamma_{1}
$$

and so

$$
V(K) \cap \Gamma=\bigcup_{\gamma \in \Gamma_{\text {tor }}} V(K) \cap\left(\gamma+\Gamma_{1}\right)=\bigcup_{\gamma \in \Gamma_{\text {tor }}}\left(\gamma+(-\gamma+V(K)) \cap \Gamma_{1}\right) .
$$

Using the fact $\Gamma_{\text {tor }}$ is finite and applying Theorem 2.5 to each intersection $(-\gamma+V(K)) \cap \Gamma_{1}$ thus completes our proof.

We use the ideas from [Ghi06b] to describe the intersection of a curve $C$ with a $\phi$-module of rank one. So, let $\left(x_{1}, \ldots, x_{g}\right) \in \mathbb{G}_{a}^{g}(K)$, let $\Gamma$ be the cyclic $\phi$-submodule of $\mathbb{G}_{a}^{g}(K)$ generated by $\left(x_{1}, \ldots, x_{g}\right)$, and let $\bar{\Gamma}$ be the $\phi$-submodule of rank one, containing all $\left(z_{1}, \ldots, z_{g}\right) \in \mathbb{G}_{a}^{g}(\bar{K})$ for which there exists a nonzero polynomial $P$ such that

$$
\phi_{P}\left(z_{1}, \ldots, z_{g}\right) \in \Gamma \text {. }
$$

Since all polynomials $\phi_{P}$ (for $P \in A$ ) are separable, we have $\bar{\Gamma} \subset \mathbb{G}_{a}^{g}\left(K^{\mathrm{sep}}\right)$.

With the notation above, we prove the following result; this may be viewed as a Drinfeld module analog of McQuillan's result on semiabelian varieties (see [McQ95]), which had been conjectured by Lang.

Theorem 4.2. Let $C \subset \mathbb{G}_{a}^{g}$ be an affine curve defined over $K$. Then $C(\bar{K}) \cap \bar{\Gamma}$ is a finite union of cosets of $\phi$-submodules of $\bar{\Gamma}$. Moreover, each $\phi$-submodule appearing in the above intersection is of the form $B_{i}(K) \cap \Gamma$ for some algebraic $\phi$-submodule $B_{i}$.

Before proceeding to the proof of Theorem 4.2 we first prove two facts which will be used later. The first fact is an immediate consequence of Theorem 1 of [Sca02] (the Denis-Manin-Mumford conjecture for Drinfeld modules), which we state below.

Theorem 4.3 (Scanlon). Let $V \subset \mathbb{G}_{a}^{g}$ be an affine variety defined over $\bar{K}$. Then there exist algebraic $\phi$-submodules $B_{1}, \ldots, B_{\ell}$ of $\mathbb{G}_{a}^{g}$ and elements $\gamma_{1}, \ldots, \gamma_{\ell}$ of $\phi_{\text {tor }}$ such that

$$
V(\bar{K}) \cap \phi_{\text {tor }}=\bigcup_{i=1}^{\ell}\left(\gamma_{i}+B_{i}(\bar{K})\right) \cap \phi_{\text {tor }} .
$$

Moreover, in [Sca02, Remark 19], Scanlon notes that his proof of the Denis-Manin-Mumford conjecture yields a uniform bound on the degree of the Zariski closure of $V(\bar{K}) \cap \phi_{\text {tor }}$, depending only on $\phi, g$, and the degree of $V$. In particular, one obtains the following uniform statement for translates of curves.

FACT 4.4. Let $C \subset \mathbb{G}_{a}^{g}$ be an irreducible curve which is not a translate of an algebraic $\phi$-module of $\mathbb{G}_{a}^{g}$. Then there exists a positive integer $N$ such that, for every $y \in \mathbb{G}_{a}^{g}(\bar{K})$, the set $(y+C(\bar{K})) \cap \phi_{\text {tor }}$ has at most $N$ elements.

Proof. The curve $C$ contains no translate of a positive-dimensional algebraic $\phi$-submodule of $\mathbb{G}_{a}^{g}$, so for every $y \in \mathbb{G}_{a}^{g}(\bar{K})$, the algebraic $\phi$-modules $B_{i}$ appearing in the intersection $(y+C(\bar{K})) \cap \phi_{\text {tor }}$ 


\section{GHIOCA AND T. J. TUCKER}

are all trivial. In particular, the set $(y+C(\bar{K})) \cap \phi_{\text {tor }}$ is finite. Thus, using the uniformity obtained by Scanlon for his Manin-Mumford theorem, we conclude that the cardinality of $(y+C(\bar{K})) \cap \phi_{\text {tor }}$ is uniformly bounded above by some positive integer $N$.

We also use the following fact in the proof of our Theorem 4.2.

FACT 4.5. Let $\phi: A \rightarrow K\{\tau\}$ be a Drinfeld module. Then for every positive integer $D$, there exist finitely many torsion points $y$ of $\phi$ such that $[K(y): K] \leqslant D$.

Proof. If $y \in \phi_{\text {tor }}$, then the canonical height $\widehat{h}(y)$ of $y$ (as defined in [Den92b]) equals zero. Also, as shown in [Den92b], the difference between the canonical height and the usual Weil height is uniformly bounded on $\bar{K}$. Then Fact 4.5 follows by noting that there are finitely many points of bounded Weil height and bounded degree over the field $K$ (using Northcott's theorem applied to the global function field $K$ ).

We are now ready to prove Theorem 4.2.

Proof of Theorem 4.2. Arguing exactly as in the proof of Theorem 2.5, we can obtain the 'moreover' clause from the main statement. Furthermore, it suffices to show that if $C$ is an irreducible affine curve (embedded in $\mathbb{G}_{a}^{g}$ ), then $C(\bar{K}) \cap \bar{\Gamma}$ is infinite only if $C$ is a translate of an algebraic $\phi$-submodule (because any translate of an algebraic $\phi$-module intersects $\bar{\Gamma}$ in a coset of some $\phi$-submodule of $\bar{\Gamma}$ ). Therefore, from now on, we assume that $C$ is irreducible, that $C(\bar{K}) \cap \bar{\Gamma}$ is infinite, and that $C$ is not a translate of an algebraic $\phi$-submodule. We will derive a contradiction.

Let $z \in C(\bar{K}) \cap \bar{\Gamma}$. For each field automorphism $\sigma: K^{\text {sep }} \rightarrow K^{\text {sep }}$ that restricts to the identity on $K$, we have $z^{\sigma} \in C\left(K^{\text {sep }}\right.$ ) (because $C$ is defined over $\left.K\right)$. By the definition of $\bar{\Gamma}$, there exists a nonzero polynomial $P \in A$ such that $\phi_{P}(z) \in \Gamma$. Since $\phi_{P}$ has coefficients in $K$, we obtain

$$
\phi_{P}\left(z^{\sigma}\right)=\left(\phi_{P}(z)\right)^{\sigma}=\phi_{P}(z) .
$$

The last equality follows from the fact that $\phi_{P}(z) \in \Gamma \subset \mathbb{G}_{a}^{g}(K)$. We conclude that $\phi_{P}\left(z^{\sigma}-z\right)=0$ and, thus, we have

$$
T_{z, \sigma}:=z^{\sigma}-z \in \phi_{\text {tor }} .
$$

Moreover, $T_{z, \sigma} \in(-z+C(\bar{K})) \cap \phi_{\text {tor }}$ (because $\left.z^{\sigma} \in C\right)$. Using Fact 4.4 we conclude that for each fixed $z \in C(\bar{K}) \cap \bar{\Gamma}$, the set $\left\{T_{z, \sigma}\right\}_{\sigma}$ has cardinality bounded above by some number $N$ (independent of $z$ ). In particular, this implies that $z$ has finitely many Galois conjugates, so $[K(z): K] \leqslant N$. Similarly we have $\left[K\left(z^{\sigma}\right): K\right] \leqslant N$; thus, we may conclude that

$$
\left[K\left(T_{z, \sigma}\right): K\right] \leqslant\left[K\left(z, z^{\sigma}\right): K\right] \leqslant N^{2} .
$$

As shown by Fact 4.5, there exists a finite set of torsion points $w$ for which $[K(w): K] \leqslant N^{2}$. Hence, recalling that $N$ is independent of $z$, we see that the set

$$
H:=\left\{T_{z, \sigma}\right\} \underset{\substack{z \in C(\bar{K}) \cap \bar{\Gamma} \\ \sigma: K^{\mathrm{sep}} \rightarrow K^{\mathrm{sep}}}}{ } \text { is finite. }
$$

Now, since $H$ is a finite set of torsion points, there must exist a nonzero polynomial $Q \in A$ such that $\phi_{Q}(H)=\{0\}$. Therefore, $\phi_{Q}\left(z^{\sigma}-z\right)=0$ for each $z \in C(\bar{K}) \cap \bar{\Gamma}$ and each automorphism $\sigma$. Hence, $\phi_{Q}(z)^{\sigma}=\phi_{Q}(z)$ for each $\sigma$. Thus, we have

$$
\phi_{Q}(z) \in \mathbb{G}_{a}^{g}(K) \text { for every } z \in C(\bar{K}) \cap \bar{\Gamma} .
$$

Let $\Gamma_{1}:=\bar{\Gamma} \cap \mathbb{G}_{a}^{g}(K)$. Since $\bar{\Gamma}$ is a finite rank $\phi$-module and $\mathbb{G}_{a}^{g}(K)$ is a tame module (i.e. every finite rank submodule is finitely generated; see [Poo95] for a proof of this result), it follows that $\Gamma_{1}$ is finitely generated. Let $\Gamma_{2}$ be the finitely generated $\phi$-submodule of $\bar{\Gamma}$ generated by all points $z \in \bar{\Gamma}$ such that $\phi_{Q}(z) \in \Gamma_{1}$. More precisely, if $w_{1}, \ldots, w_{\ell}$ generate the $\phi$-submodule $\Gamma_{1}$, then for 


\section{A DYNAMicAl VERSion OF THE MORDELL-LANG CONJECTURE For THE ADDITIVE GROUP}

each $i \in\{1, \ldots, \ell\}$, we find all of the finitely many $z_{i}$ such that $\phi_{Q}\left(z_{i}\right)=w_{i}$. Then this finite set of all $z_{i}$ generate the $\phi$-submodule $\Gamma_{2}$. Thus, $\Gamma_{2}$ is a finitely generated $\phi$-submodule and, moreover, using (4.5.3), we obtain $C(\bar{K}) \cap \bar{\Gamma}=C(\bar{K}) \cap \Gamma_{2}$. Since $\Gamma_{2}$ is a finitely generated $\phi$-submodule of rank one (because $\Gamma_{2} \subset \bar{\Gamma}$ and $\bar{\Gamma}$ has rank 1), Theorem 4.1 finishes the proof of Theorem 4.2.

\section{REFERENCES}

Bak75 A. Baker, Transcendental number theory (Cambridge University Press, Cambridge, 1975).

Bos99 V. Bosser, Minorations de formes linéaires de logarithmes pour les modules de Drinfeld, J. Number Theory 75 (1999), 279-323.

BS66 A. I. Borevich and I. R. Shafarevich, Number theory (Translated from the Russian by Newcomb Greenleaf), Pure and Applied Mathematics, vol. 20 (Academic Press, New York, 1966).

Cha41 C. Chabauty, Sur les points rationnels des courbes algébriques de genre supérieur à l'unité, C. R. Math. Acad. Sci. Paris 212 (1941), 882-885.

Col85 R. F. Coleman, Effective Chabauty, Duke Math. J. 52 (1985), 765-770.

Dav95 S. David, Minorations de formes linéaire de logarithmes elliptiques, Mém. Soc. Math. France (N.S.) vol. 62 (1995).

Den92a L. Denis, Géométrie diophantienne sur les modules de Drinfel'd, in The arithmetic of function fields, Columbus, OH, 1991, Ohio State University Mathematical Research Institution Publications, vol. 2 (de Gruyter, Berlin, 1992), 285-302.

Den92b L. Denis, Hauteurs canoniques et modules de Drinfel'd, Math. Ann. 294 (1992), 213-223.

Fal94 G. Faltings, The general case of S. Lang's conjecture, in Barsotti Symposium in Algebraic Geometry, Abano Terme, 1991, Perspectives in Mathematics, No. 15 (Academic Press, San Diego, CA, 1994), 175-182.

Ghi05 D. Ghioca, The Mordell-Lang theorem for Drinfeld modules, Int. Math. Res. Not. 53 (2005), 3273-3307.

Ghi06a D. Ghioca, Equidistribution for torsion points of a Drinfeld module, Math. Ann. 336 (2006), 841-865.

Ghi06b D. Ghioca, Towards the full Mordell-Lang conjecture for Drinfeld modules, Preprint (2006), arXiv:math/0611476.

GT D. Ghioca and T. J. Tucker, Equidistribution and integral points for Drinfeld modules, Trans. Amer. Math. Soc., to appear.

GT07a D. Ghioca and T. J. Tucker, Siegel's theorem for Drinfeld modules, Math. Ann. 339 (2007), 37-60.

GT07b D. Ghioca and T. J. Tucker, p-adic logarithms for polynomial dynamics, Preprint (2007), arXiv:0705.4047.

GT07c D. Ghioca and T. J. Tucker, Mordell-Lang and Skolem-Mahler-Lech theorems for endomorphisms of semiabelian varieties, Preprint (2007), arXiv:0710.1669v1.

GTZ D. Ghioca, T. J. Tucker and M. E. Zieve, Intersections of polynomial orbits, and a dynamical Mordell-Lang conjecture, Invent. Math., to appear.

Gos96 D. Goss, Basic structures of function field arithmetic, Ergebnisse der Mathematik und ihrer Grenzgebiete (3), vol. 35 (Springer, Berlin, 1996).

Hir91 N. Hirata-Kohno, Formes linéaires de logarithmes de points algébriques sur les groupes algébriques, Invent. Math. 104 (1991), 401-433.

Hru96 E. Hrushovski, The Mordell-Lang conjecture for function fields, J. Amer. Math. Soc. 9 (1996), 667-690.

Hru98 E. Hrushovski, Proof of Manin's theorem by reduction to positive characteristic, in Model theory and algebraic geometry, Lecture Notes in Mathematics, vol. 1696 (Springer, Berlin, 1998), 197-205.

McQ95 M. McQuillan, Division points on semi-abelian varieties, Invent. Math. 120 (1995), 143-159. 
A Dynamical Version of the Mordell-Lang CONJecture For the AdDitive Group

Poo95 B. Poonen, Local height functions and the Mordell-Weil theorem for Drinfeld modules, Compositio Math. 97 (1995), 349-368.

Sca02 T. Scanlon, Diophantine geometry of the torsion of a Drinfeld module, J. Number Theory $\mathbf{9 7}$ (2002), 10-25.

Ser97 J.-P. Serre, Lectures on the Mordell-Weil theorem, third edition, Translated from the French and edited by Martin Brown from notes by Michel Waldschmidt, with a foreword by Brown and Serre, Aspects of Mathematics (Vieweg, Braunschweig, 1997).

SUZ97 L. Szpiro, E. Ullmo and S. Zhang, Equirépartition des petits points, Invent. Math. 127 (1997), 337-347.

Voj96 P. Vojta, Integral points on subvarieties of semiabelian varieties. I, Invent. Math. 126 (1996), $133-181$.

Zha98 S. Zhang, Equidistribution of small points on abelian varieties, Ann. of Math. (2) 147 (1998), 159-165.

Zha06 S. Zhang, Distributions and heights in algebraic dynamics, A tribute to Prof. S.-S. Chern, Survey in Differential Geometry, vol. 10 (International Press, Boston, MA, 2006), 381-430.

D. Ghioca dragos.ghioca@uleth.ca

Department of Mathematics and Computer Science, University of Lethbridge, 4401 University Drive, Lethbridge, AB, Canada T1K 3M4

T. J. Tucker ttucker@math.rochester.edu

Department of Mathematics, Hylan Building, University of Rochester, Rochester, NY 14627, USA 